\title{
Bilingual Information Hiding System: A Formalized Approach
}

\author{
${ }^{1}$ Riad Jabri, ${ }^{2}$ Boran Ibrahim \\ Faculty of Information Technology, Computer Science Department, \\ University of Jordan, Amman, Jordan \\ jabri@ju.eu.jo; boranabed@yahoo.com
}

\begin{abstract}
Steganography and cryptography are used to maintain privacy and security over communication channels. Due to their complexity and diversity, there is a need for their continuous improvements. In this paper, we consider such improvements and propose a new bilingual information hiding system. The proposed system is based on a formal approach that incorporates text-based steganography and cryptography in a way that permits multilayered levels of security and privacy and improves the quality of information hiding in terms of perceptual transparency, robustness and hiding capacity. Considering an Arabic text as a cover one, the inter-word spaces and word features have been used as places for information hiding. As a result, 3.676 have been achieved as an improved average of capacity ratio.
\end{abstract}

Keywords: Information hiding; Steganography; Cryptography; Encoding; Decoding.

\section{Introduction}

Steganography is an information hiding technique [1, 2, 3]. For the purpose of our research, we consider steganography as a method of hiding the existence of a bilingual secret message in Arabic text [4]. Textsteganography proceeds according to the following scheme [5]:

- A secret message is concealed in a cover- text using an embedding algorithm to produce a stego- text.

- The stego - text is then transmitted over a communication channel (Internet).

- Upon its delivery, the secret message is recovered using an extracting algorithm.

- $\quad$ The embedding and the extracting algorithms are augmented by the so called a stego- key to encrypt and decrypt the hidden data respectively.

Based on the presented scheme, the secret message is concealed using one of the following methods [5, 6]:

- Modification of the cover-text, such as insertion of spaces, misspelling, modifying the features (name, shape, position, color, size) of the individual characters

- Substitution, such as replacement of insignificant data within the cover text by hidden ones.

- Generation, such as creation of a fake cover.

The most recent efforts, techniques and tools related to our work can be classified as follows: 
- Approaches based on inter-word and inter-paragraph spacing to generate dynamic stegotext, as suggested by Por et. al. [7] and Jabri et. al. [5].

- Techniques based on combining the following methods: Open space; syntactic (punctuation) and semantic encoding (synonym words), as suggested by Bender et al. [2].

- Tools based on open space concealing method combined with compression and encryption, such as SNOW as suggested by Kwan [8].

- $\quad$ Technique based on natural language processing and using either the sentence structures or linguistic coding scheme, as suggested by Bergmair [9].

- Techniques specialized for Arabic text, classified into four categories [10 ]:

1. Dot steganography defined as a vertical displacement of dots in Arabic characters [11].

2. La steganography uses special forms of " La" word for hiding information [12]

3. Techniques that use letters with extension (kashida) and un-extended ones to hold the secret bits "one" and "zero" respectively [13, 14].

4. Techniques that use a diacritic Arabic text for information hiding [15].

The above-mentioned efforts aim at improving one or more of the following quality indictors: perceptual transparency, robustness and hiding capacity [15]. For example, the hiding algorithms suggested in $[13,14]$ aim at improving hiding capacity.

Our proposed approach constitutes further improvements of such indictors. In addition, it is characterized by the following:

- The system hides bilingual (Arabic, English) secret messages. It combines inter-word spaces and letter extension (kashida) to hide secret bits. Such combination contributes to perceptual transparency, robustness and increases the hiding capacity.

- The system is based on a formal approach that combines steganography and cryptography as function compositions and introduces multilayered security levels.

The remainder of this paper is organized as follows. Section 2 presents formalization and implementation of the proposed system. Section3 presents analysis and results. A conclusion is given in section 4.

\section{The Proposed Method}

Based on our set objectives, we define a steganography system in terms of two main stages. The first one is an encoding stage to embed a secret message in a cover text and produce a stego-text. The second one is a decoding stage to extract the secret message from the stego text. The encoding stage transforms the secret message into a sequence of encrypted bits. Such transformation improves the efficiency, the privacy and the security of the hiding process. Then, it considers the cover text as composing of sequence of characters and the uses these characters as positions to hide the secret bits. Such a use is based on the fact that most of Arabic letters can be extended and such extension is considered as one of its writing styles. Thus, the hiding positions are defined as spaces and letters suitable for extension. The spaces are extended by additional space and the letters are extended by kashida (-) Hence, the Arabic characters are classified into two sets. The first one includes the Arabic characters that are suitable for extension by kashida. For example, the letters " ص and "' are 
members of such a set. They can be extended by kashida as "صـ " " " " respectively. The second set includes the special characters (?, : , ", \%), the digits $(0, . ., 9)$ and the non-extendable Arabic characters . For example, the letters"j" , و" و" ر" ر" .

Based on such classification, suitability criteria are defined and used to determine the hiding positions in the cover text. The hiding process then proceeds as follows. The secret bits and the cover text are scanned bit by bit and character by character respectively. Upon capturing a hiding position, the respective character (blank or extendable letter) is extended, if the secret bit is "1". Otherwise, no extension is performed. On the other hand, the decoding stage includes an extracting process to retrieve the hidden bits from their respective positions in the stego-text.

Based on the above-mentioned definition, the encoding and the decoding stages are formalized as a composition of respective functions. The implementation of the proposed system is then reduced to the implementation of an interaction context and the functions from which these stages are composed. They have been implemented according to the algorithms given below using C\#.NET as a programming tool. As a result, the proposed system has been constructed with following functionality:

- The sender interacts with the proposed system through an interaction context to facilitate: User authentication; browsing the secret message (SM) and the cover text (CT) from their respective text files; testing the suitability of CT to hide SM and initiating the encoding process.

- In addition to authentication, the receiver interaction context facilitates: browsing the stegotext from its respective text file; and initiating the decoding process.

- The system responds to the sender-initiated request by activating the functions respective to the encoding stage.

- The system responds to the receiver-initiated request by activating the functions respective to the encoding stage.

\subsection{The encoding stage}

The encoding stage consists of two major steps. First, it transforms the secret message into a sequence of encrypted bits. Second, it considers the cover text as composing of sequence of characters and the uses these characters as positions to hide the secret bits. These steps are formalized and implemented as follows.

Let $\mathrm{SM}=\mathrm{SM}_{1} \mathrm{SM}_{2} \ldots \mathrm{SM}_{\mathrm{n}}$ be the sequence of characters representing the secret message.

The transformation step is defined as a composition of the functions:

Bit (Encrypt (Compress (Byte (SM[ ] )) $\rightarrow$ BSM[ ], where

- Byte (SM[ ]) $\rightarrow \mathrm{SM}[]$ is a function that converts the secret message (SM) to a stream of bytes, using Unicode encoding.

- Compress (SM[ ]) $\rightarrow$ SM[ ] is a compression function, for example, eliminating extra zerobytes in the Unicode for Arabic.

- Encrypt (SM[ ]) $\rightarrow$ ESM[ ] is an encryption function. It uses two passwords of type string to generate a fixed random stream of keys (Key [ ]). The keys are then XORed with SM [ ] to obtain an encrypted secret message.

- $\operatorname{Bit}(\operatorname{ESM}[$ ]) BSM[ ] is function that converts the encrypted SM into a stream of bits represented as $\mathrm{BSM}[]=\mathrm{BSM}_{1} \mathrm{BSM}_{2} \ldots \mathrm{BSM}_{n}$. 
Riad Jabri, Boran Ibrahim, Haruhiko Kimura; Bilingual Information Hiding System: A Formalized Approach. Transactions on Machine Learning and Artificial Intelligence, Volume 3 No 3 June (2015); pp: 33-45

Let $\mathrm{EC}=\{ت, \ldots, \varphi\}$ denotes the set of extendable Arabic characters.

Let NEC $=\{9 \ldots, 0,1, \ldots, j, j\}$ denotes the set of non-extendable Arabic characters.

Let $P E C=\left\{(A C i, A C j)\right.$ denotes the set of pair-wise Arabic characters $\left(A C_{i}, A C_{j}\right)$ such that $A C_{i} E C, A C_{j}(E C$ or NEC) and their occurrence as sequence in a text enables the extension of $A C_{i}$.

A hiding position is defined as an Arabic character (AC) subject to the following suitability criteria

- $\quad$ Suitability $(A C)=$ True, if $(A C \quad($ NEC PEC) or $A C="$ ")

- $\quad$ Suitability $(A C)=$ False, otherwise.

Let $\mathrm{CT}=\mathrm{CT}_{1} \mathrm{CT}_{2} \ldots \mathrm{CT}_{\mathrm{n}}$ is the sequence of characters representing the cover text.

Let HidingPosition (CT[ ]) $\rightarrow \mathrm{HCT}[$ ] be a function to determine the hiding positions in the cover text.

The embedding process is then defined by the function Embed (BSM, HCT) $\rightarrow$ ST. However, such embedding is subject to satisfied quality indicators computed by the following functions:

- HidingRatio to express conceivability as (Length (BSM)/Length (hiding positions))\%

- CapacityRatio to express conceivability as ( Bytes( SM)/Bytes(CT))\%

Thus, the encoding stage proceeds according to following steps:

Step1: SM = Input (Secret-message);

Step2: BSM[ ] = Bit (Encrypt (Compress (Byte (SM)))

Step3: CT = Select-cover-text

Step4: HCT [ ] = HidingPosition (CT[ ])

Step4: Compute quality indicators

Step3: If (Satisfied (quality indicators) $\{$ Embed ( BSM, HCT)\}

Else if $\{$ repeat from step3\}

The implementation of the encoding stage is reduced to the implementation of its functions according to respective algorithms. Representative ones are given below.

\section{Algorithm 1: Encryption and Decryption}

Input: compressed stream of bytes respective to SM

Output: encrypted or decrypted stream of bytes respective to SM

Method:

encryptedMessage [ ] = encrypt (newMessage, password1) ;

decryptedMessage [ ] = decrypt (encryptedMessage, password2) ;

encrypt (message [ ] , password1)

\{

return EncryptDecrypt (message, password1) ; 
decrypt (message [ ] , password2)

\{

return EncryptDecrypt (message, password1);

\}

EncryptDecrypt (message [ ] , password)

\{

randomNumbers [ ] $=\{08,06,02\}$;

DerivePasswordBytes $=\mathrm{dpb}$ (password, randomNumbers) ;

key [ ] =dpb.GetBytes(128) ; // Return 128 random numbers

returnMessage [ message. length ] ;

for $\mathrm{i}=1$ To message. length step 1

\{

index $=\mathrm{i}$ mod key. length ;

returnMessage [i ] = key [index] XOR message [i ] ;

\}

return returnMessage ;

\}

Algorithm 1 performs encryption and decryption. It uses two passwords of type string to generate a fixed random stream of keys The keys are then XORed with SM (or CT)to obtain an encrypted (or decrypted) secret message. By using passwords and randomly generated keys, Algorithm 1 introduces two levels of security.

\section{Algorithm 2: Hiding positions}

Input: Covertext (CT)

Output: Hiding positions in CT.

Method:

Hiding positions [ ] = Suitable $(\mathrm{CT}[\mathrm{]})$

Suitable (CT[ ] )

$\{$ for $\mathrm{i}=1$ To $\mathrm{CT}$. length step 1

\{

if ( Suitability (CT [i] = True ) Then

$\{$ HidingPositions [i ] = " 1"

Else

$\{$ HidingPositions $[i]=$ " $0 "$

\} 


\section{Algorithm 3: Embedding}

Input: The stream of bits BSM respective to the secret message SM.

The over text (CT)

The hiding positions (HidingPositions) in CT

Output: stego_text (secret bits inside cover text).

Method:

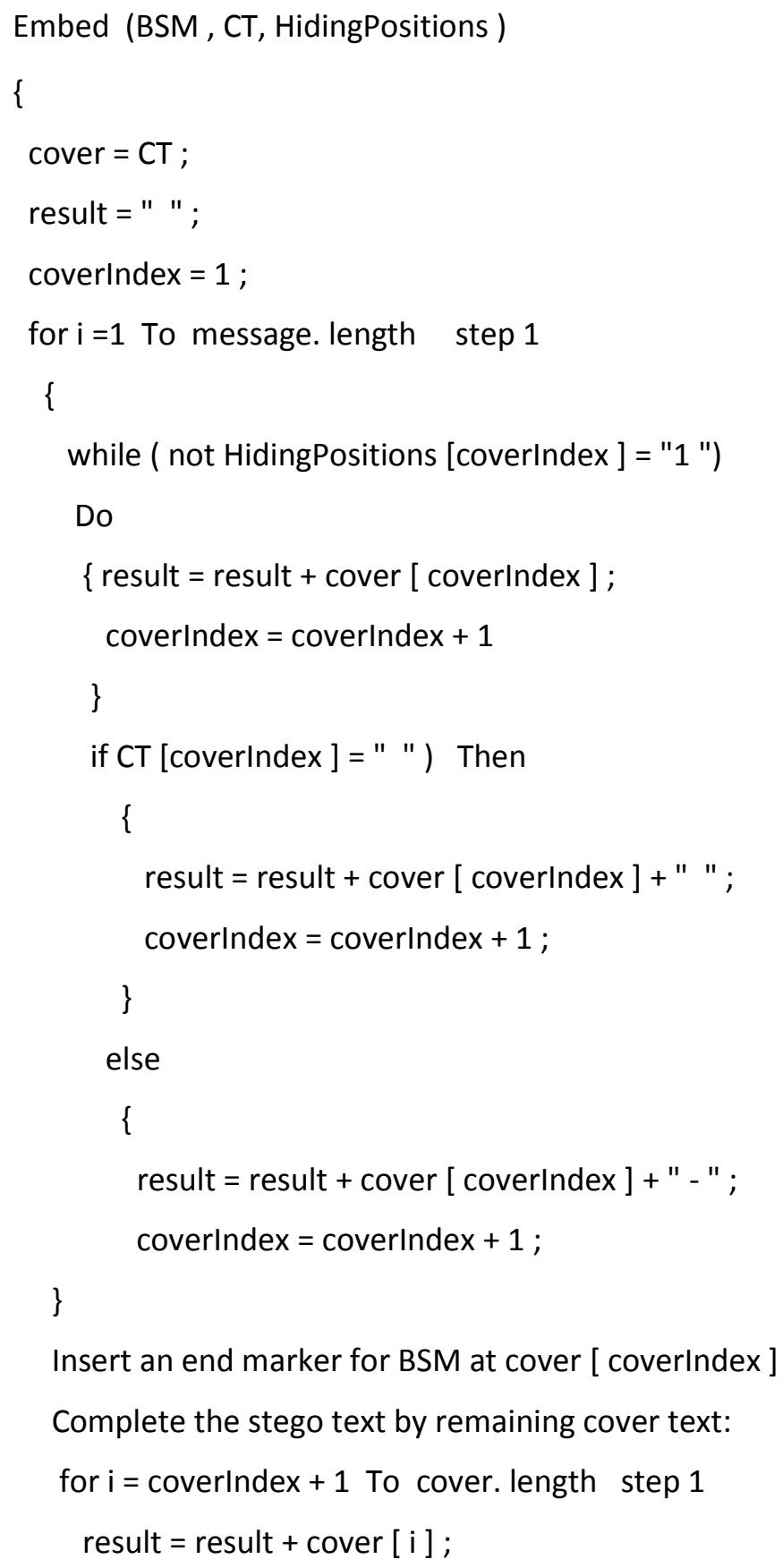


return result ;

\} .

\subsection{The Decoding Stage}

The decoding stage extracts the secret message from the stego-text at receiver's side according to the following steps:

Step 1: ST = Browse (stego-text)

Step 2: If (authentication $=$ True) $\{$ Decompress $($ Decrypt $($ Byte $($ Bit-extract $(\mathrm{ST})))\}$, where

- Bit-extract (ST) BSM[ ] is function that extracts the encrypted SM as the stream of bits $\mathrm{BSM}\left[\mathrm{]}=\mathrm{BSM}_{1} \mathrm{BSM}_{2} \ldots \mathrm{BSM}_{\mathrm{n}}\right.$

- Byte (BSM[ ] ) SM[ ] is a function that converts extracted message (BSM) to a stream of bytes, represented as SM[ ]

- Decrypt (SM[ ]) SM[ ] XOR Key [ ] is an decryption function. As in Encrypt, the function Decrypt uses two passwords of type string to generate a fixed random stream of keys. However, the keys are XORed with SM [ ] to obtain a decrypted secret message.

- Decompress (SM[ ]) SM[ ] is a decompression function to recover SM as represented by Unicode for Arabic.

The implementation of the decoding stage is reduced to an interaction context for authentication, browsing the stego text and then displaying the secret message. This is achieved by applying the functions respective to the decoding stage. These functions have been implemented according to respective algorithms. Representative ones are given below.

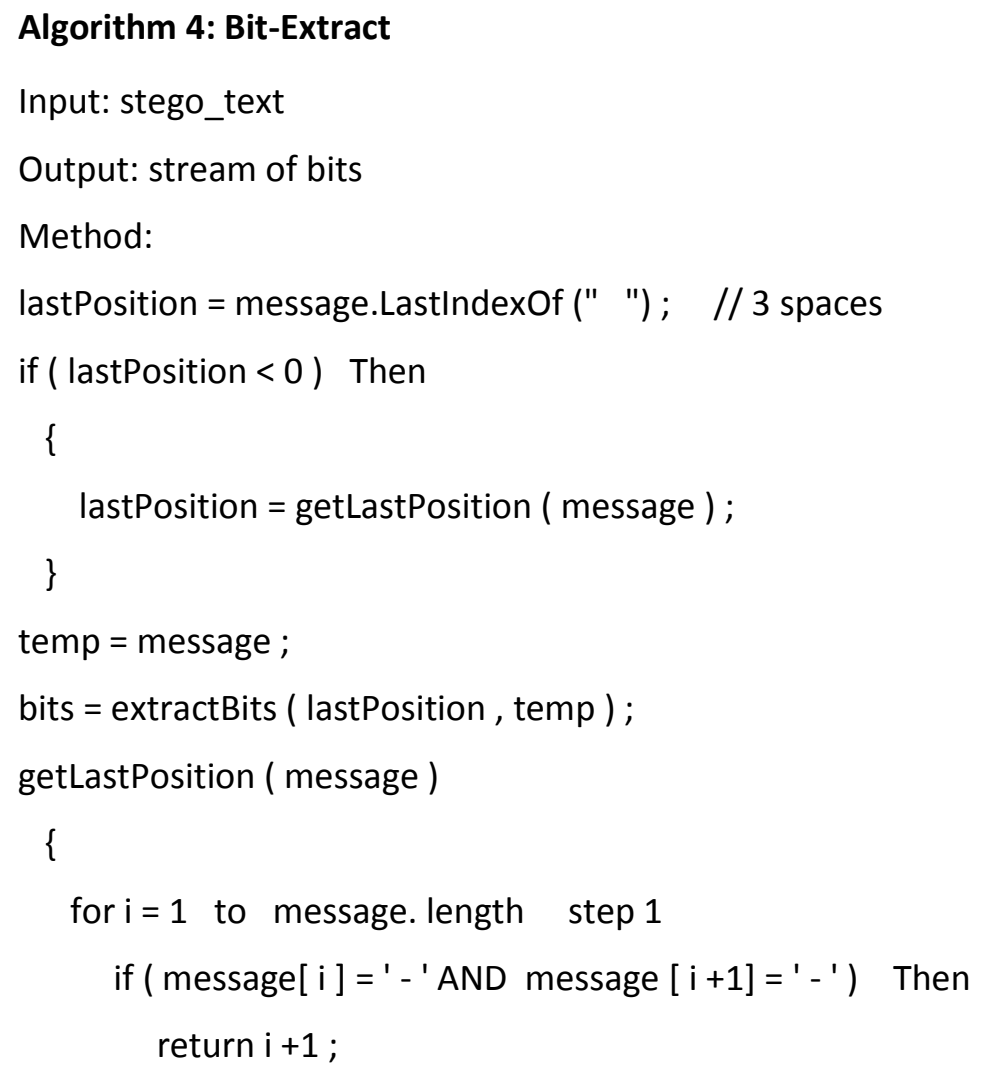


return i ;

\}

extractBits ( lastPosition , temp )

\{

bits = " " ;

for $\mathrm{i}=0$ To lastPosition step 1

if ( temp [i]= ' ' ) Then

\{

if ( temp $[\mathrm{i}+1]={ }^{\prime}$ ' $)$ Then

\{

bits = bits + " $1 "$;

$\mathrm{i}=\mathrm{i}+1$;

\}

else

bits = bits + " 0 ";

\}

else if ( temp [i ] = ' - ') Then

\{

bits = bits + " $1 "$;

\}

else

\{

if ( temp $[i+1]<>$ ' - ') Then

if checkTwoCharacters ( temp [i], temp $[i+1])$ ) Then

\{

bits = bits + " 0 " ;

\}

\}

return bits ;

\} .

Algorithm 5: Conversion to bytes

Input: stream of bits

Output: stream of bytes

Method: 
message [ ] = decode (bits);

decode (bits)

\{

hidden [bits. length / 8] ;

$c=0$;

for $i=1$ To hidden. length step 1

for $\mathrm{j}=7$ To 0 step -1

\{

if (bits $[\mathrm{c++}]=$ ' 1 ') Then

hidden $[\mathrm{i}]=($ hidden $[\mathrm{i}]$ OR $(1<<j))$;

\}

return hidden ;

\}.

\section{Experiments and Analysis}

The proposed system has been developed with an interface represented by two forms. The first form is denoted facilitates interaction with the presented encoding stage and its respective concealing functions. Furthermore, it displays quality indicators such as hiding and capacity ratios respective to the secret message and the browsed cover text. The second form facilitates interaction with the presented decoding stage and its respective functions. Through its interface, the proposed steganography system has been tested using several multilingual texts (Arabic and English) as demonstrated by Figure 1, Figure 2, Figure 3 and Figure 4 respectively.

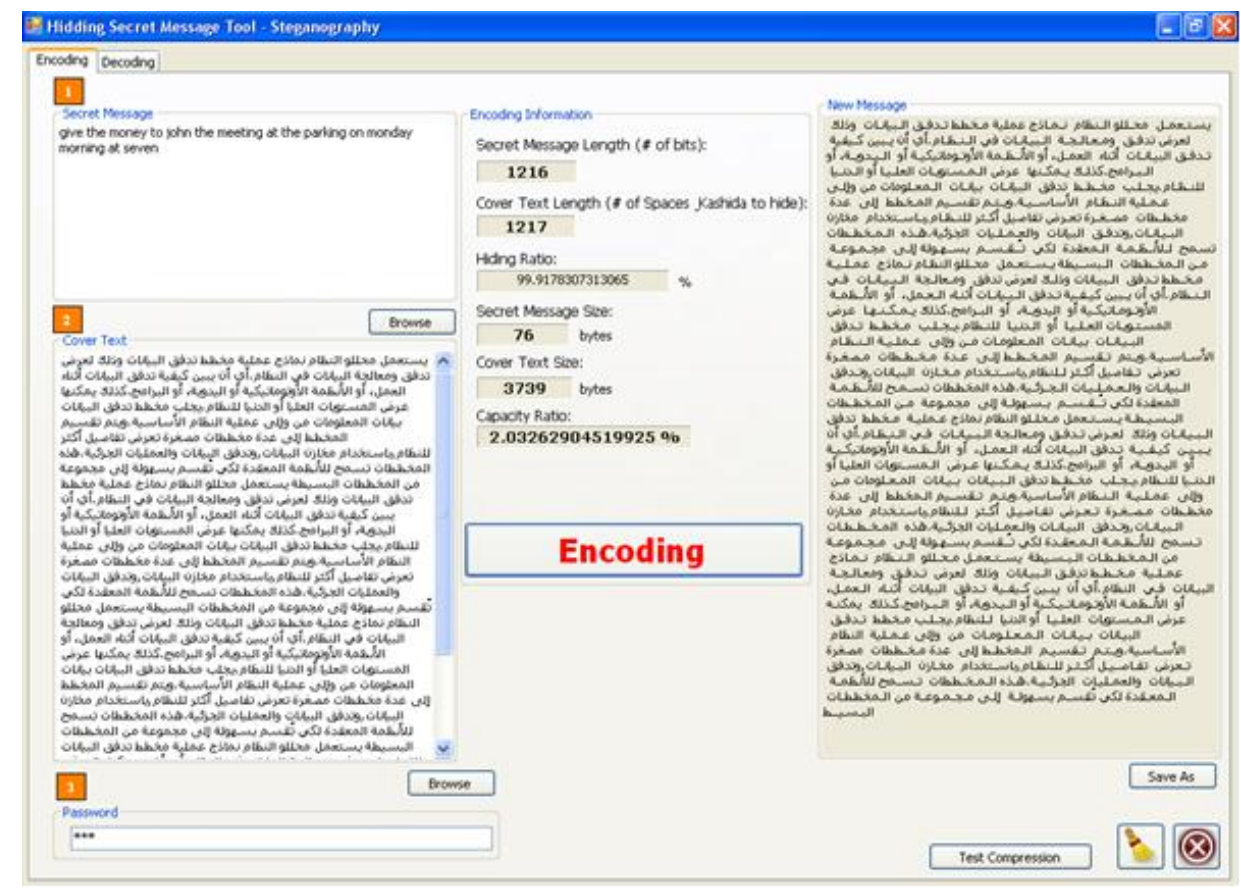

Figure 1: Encoding of English text 


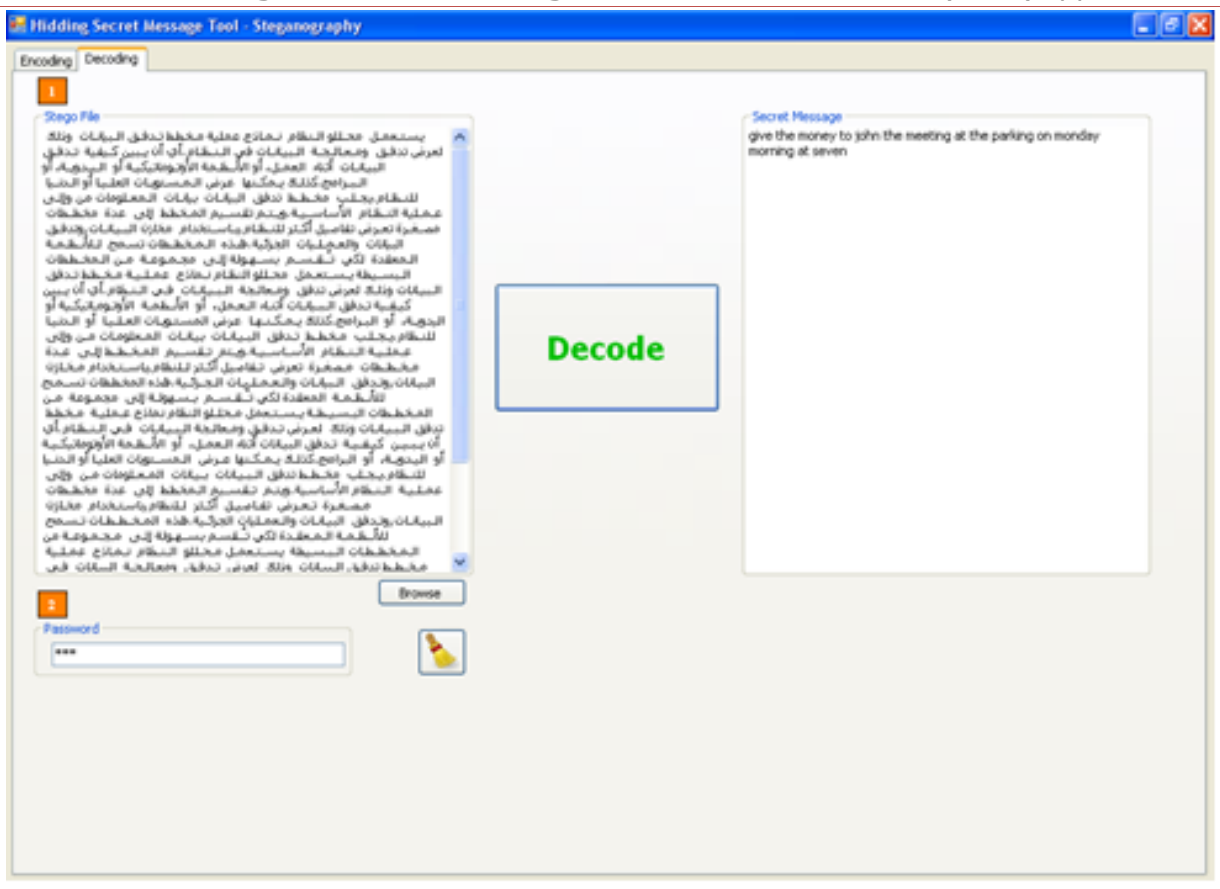

Figure 2: Decoding of English text

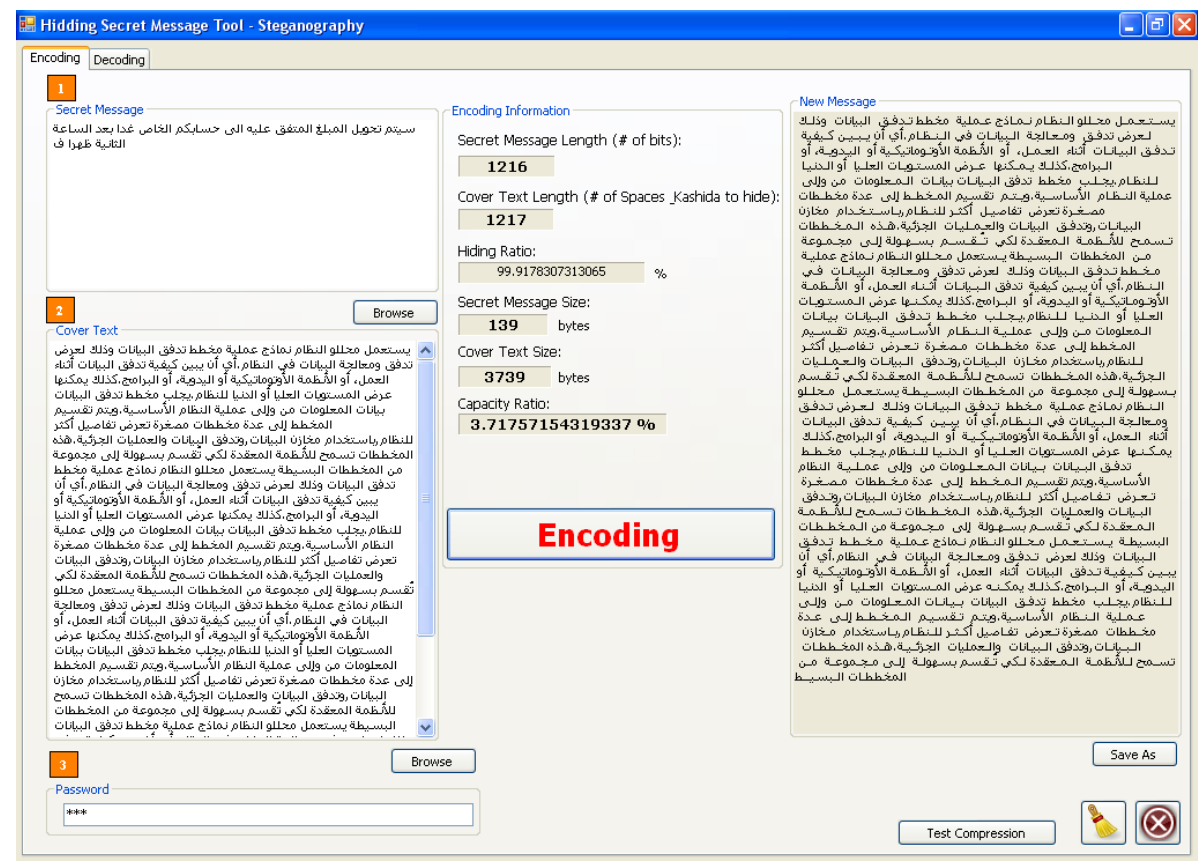

Figure 3: Decoding of an Arabic text

Table 1 shows the achieved capacity ratios for secrete messages and cover texts of different size, where 3.67 is indicated as the average capacity ratio. This ratio is higher than the ones reported by other approaches as demonstrated by table 2 . In [1], 2.46 and3.73 are reported as the average capacity ratio using one and three kashidas respectively. However, our approach is based on using one kashida per letter. Using the same text as a cover one, Table 3 shows the size of the stego_text for Arabic and 
English secrete messages. In both cases the size of cover text has been increased by $27.6 \%$ and $29 \%$ respectively.

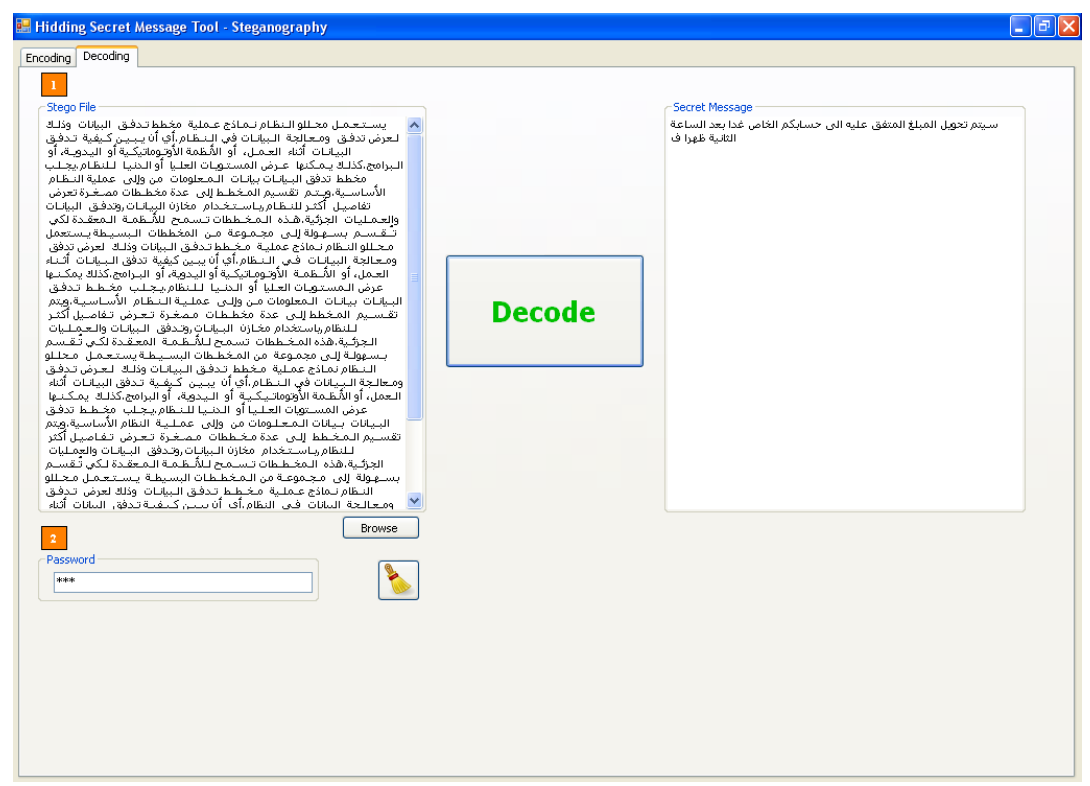

Figure 4: Decoding of an Arabic text

Table 1: The achieved capacity ratio

\begin{tabular}{|c|c|c|c|}
\hline $\begin{array}{c}\text { Cover size } \\
\text { (byte) }\end{array}$ & $\begin{array}{c}\text { Number of } \\
\text { hidden bits }\end{array}$ & $\begin{array}{c}\text { Capacity ratio } \\
\text { (\%) }\end{array}$ & $\begin{array}{c}\text { Average } \\
\text { capacity (\%) }\end{array}$ \\
\hline 1714 & 560 & 3.676 & 3.657 \\
\hline 6976 & 2288 & 3.655 & \\
\hline 19264 & 6320 & 3.649 & \\
\hline 31552 & 10352 & 3.648 & \\
\hline
\end{tabular}

Table 2: The capacity ratios achieved by other approaches

\begin{tabular}{|c|c|}
\hline Approach & Capacity ratio (\%) \\
\hline Dots[15 ] & 1.37 \\
\hline Kashida [13 ] & $2.46-3.73$ \\
\hline Kashida [14 ] & 3.09 \\
\hline Diactrics [15 ] & 3.27 \\
\hline
\end{tabular}

Table 3: The size of the secret message

\begin{tabular}{|c|c|c|}
\hline $\begin{array}{c}\text { Type of the } \\
\text { secret message }\end{array}$ & $\begin{array}{c}\text { Size of the } \\
\text { cover text }\end{array}$ & $\begin{array}{c}\text { Size of the } \\
\text { stego_text }\end{array}$ \\
\hline Arabic & $3.65 \mathrm{~kb}$ & $4.66 \mathrm{~kb}$ \\
\hline English & $3.65 \mathrm{~kb}$ & $4.71 \mathrm{~kb}$ \\
\hline
\end{tabular}

In addition to its efficiency and flexibility, the proposed text steganography system has demonstrated improvements in its quality indicators as follows: 
Riad Jabri, Boran Ibrahim, Haruhiko Kimura; Bilingual Information Hiding System: A Formalized Approach. Transactions on Machine Learning and Artificial Intelligence, Volume 3 No 3 June (2015); pp: 33-45

- The adopted encoding methods are characterized by their flexibility and enables hiding dynamic secret messages. The binary conversion and the encryption constitute two level of security

- The adopted embedding criteria combine suitability and randomness to ensure robustness of the stego-text, perceptual transparency and improved hiding capacity.

Compared to similar systems, our system has a higher increase in the size of the cover text. For example, the average size according to our system is $25 \%$ and the one according to the system proposed in [5] is $2 \%$. However, the proposed system has better results in terms of the following:

- A higher utilization percentage of the cover- text. This demonstrated by the ratio between the size of the secret message and the one of the cover text. For example, our approach has achieved 1:3 ratio while in [7] a cover text with a size $<16 \mathrm{~KB}$ is required for a secret message with a length $<4 \mathrm{~KB}$.

- A higher hiding capacity as demonstrated by Table 1 and Table 2.

\section{Conclusion}

In this research, an information hiding model has been suggested. Based on such model, a text steganography system has been implemented. The system is characterized by its scalability and flexibility. Although the proposed system has better quality indicators than the ones for similar system, more improvements are needed for such indicators. Mainly, the capacity of the cover- text and the robustness of the stego- text. Hence, efforts in this direction constitute a future work.

\section{REFERENCES}

[1]. Bennett, K., Linguistic steganography: Survey, analysis and robustness concerns for information hiding in text. Purdue University, CERIAS Technical Report, 2004.

[2]. Bender, S., Gruhl, D., Morimoto, N., Techniques for data hiding. IBM System Journal, 1996. 35(4): p. 313-336.

[3]. Komal, P., Sumit, V., Hitesh, C., A Survey of Information Hiding Techniques. International Journal of Emerging Technology and Advanced Engineering, 2013. 3(1): p.347-349

[4]. Cachin, C., An information -theoretic model for steganography. Lecture Notes in Computer Science, 1998. 1225: p. 306-318.

[5]. Jabri, R., Ibrahim, B., Al-Zoubi, H., Information Hiding: A Generic Approach. Journal of Computer Science, 2009. 5(12): 930-936.

[6]. Sumathi, G., Santanam, T., Umamaheswari, G., A study of Various steganograrphic Techniques Used for Information Hiding. International Journal of Emerging Technology and Advanced Engineering, 2013. 4(6): P. 9-25. 
[7]. Por, L., Delina, B., Information hiding: A new approach in text steganography. $7^{\text {th }}$ WSEAS Int. Conf. on Applied Computer \& Applied Computational Science, Hanghou, Chiana, 2008. P. 689-695.

[8]. Kwan, M., The SNOW homepage, http://www.darkside.com.au/snow/, 1998.

[9]. Haddouch Bergmair, R., Towards linguistics stganogrphy: A systematic investigation of approaches, Systems and Issues. Technical Report. 2004.

[10]. Shirali-Shareza, M.,H., Shirali-Shareza, M. Steganography in Persian and Arabic Unicode Texts Using Pseudo-Space and Pseudo-Connection Characters. Journal of Theoretical and Applied Information, 2008. 4( 8): p. 682-687.

[11]. Shirali-Shareza, M., H., Shirali-Shareza, M. A New Approach to Persian/Arabic Text Steganography. $5^{\text {th }}$ IEEE/ACIS on Computer and Information Science (ICIS- COMSAR 06), 2006. P. 310-315.

[12]. Shirali-Shareza, M. A New Persian/Arabic Text Stegonography Using " La Word". Proceedings of the International Joint Conference on Computer, Information and Systems Sciences and Engineering, Bridgeport, CT, USA, 2007.

[13]. Al Haidari, F., Gutub, A., Al-Kahsah, K., Hamodi, J. 2009. Improved Security and Capacity for Arabic Text Steganography Using 'Kashida' Extensions. IEEE/ACS International Conference on Computer Systems and Applications, Rabat, 2009. P. 396-399.

[14]. Al-Azawi, A.F., Fadhil, M.,A., Arabic Text Steganography Using Kashida Extensions with Huffman Code. Journal of Applied Sciences 2010. 10(5): P. 436-439.

[15]. Gutub, A., Elarian, Y., Awaideh, S., Alvi, A., Arabic Text Steganography Using Multiple Diacritics, WoSPA $5^{\text {th }}$ IEEE International Workshop on Signal Processing and its Applications, Sharjah, UAE, 2008. 